\title{
Pulmonary Alveolar Proteinosis in an Infant
}

\author{
PAUL S. SYMCHYCH* and D. M. FLYNN \\ From the Departments of Morbid Anatomy and Paediatrics, \\ The Hospital for Sick Children, Great Ormond Street, London
}

In 1958 Rosen, Castleman, and Liebow described 27 patients with a newly-recognized pulmonary disease which they called pulmonary alveolar proteinosis (PAP). Since then approximately 100 cases have been reported. These are reviewed by Davidson and Macleod (1969). Only once has this condition been reported in early infancy.

Wilkinson, Blanc, and Hagstrom (1968) described two babies who presented with respiratory symptoms, and died at $4 \frac{1}{2}$ and 3 months. The radiographic changes of PAP were present in both instances. We present a further case which differed in presenting with frequent pyogenic infections from the age of 2 weeks, but no clinical signs of respiratory disease until shortly before death at $3 \frac{1}{2}$ months. Chest $x$-rays showed abnormalities from the age of 6 weeks, but even in retrospect these could not be considered diagnostic.

\section{Case History}

The patient was the only child of healthy parents. Pregnancy and delivery were normal. Birthweight was $3.8 \mathrm{~kg}$. At 2 weeks of age, he developed profusely purulent, but sterile conjunctivitis. Despite therapy with neomycin-bacitracin eye ointment and oral cloxacillin, the conjunctival oedema and infection became worse and the cornea became obscured. At 7 weeks he was admitted to another hospital because of the conjunctivitis. Additional findings included resolving oral moniliasis and superficial skin sepsis. Weight gain had been normal, to $4.5 \mathrm{~kg}$. Bacterial cultures were again negative, as were cytology and complement-fixation tests for herpes. The conjunctivitis improved on framycetin eye drops and intramuscular ampicillin and cloxacillin, but the corneae were already scarred.

As the eyes improved, diarrhoea and vomiting with concomitant weight loss developed. The chest $x$-ray showed consolidation of the right upper lobe.

At 11 weeks he was transferred to this hospital. His weight was $3.7 \mathrm{~kg}$. He had persisting conjunctivitis, vomiting and diarrhoea, and skin infections. A

Received May 21, 1969.

* Present address: Department of Pathology, Hospital for Sick Children, Toronto, Ontario, Canada. blood culture grew Esch. coli and coagulase-negative staphylococci, while cultures from eyes and skin grew coagulase-positive staphylococci. The CSF was normal. Conjunctival scrapings for trachoma inclusion conjunctivitis virus were negative. Despite intensive systemic and local antibiotics and intramuscular $\gamma$ globulin, he continued to deteriorate, developing staphylococcal abscesses which responded slowly to therapy. The vomiting and diarrhoea which began at 9 weeks became increasingly severe, necessitating almost continuous intravenous therapy until death. No oral diet was satisfactory, and weight loss continued to $3.5 \mathrm{~kg}$. terminally.

He showed no clinical signs of respiratory disease until three days before death, when there was an increase in respiratory rate to $55 / 60$ per minute. Pyrexia and tachycardia were not present at any time. He died at 14 weeks.

Investigations. A chest $x$-ray at 6 weeks showed right upper lobe consolidation. On admission to hospital at 11 weeks, there was patchy shadowing which was extensive in the right upper zone, and less marked in the left hilar and basal regions. At 13 weeks there was generalized but less severe patchy shadowing, with an air bronchogram effect in the upper zones. There was also some prominence of bronchial line shadows suggesting thickening of the interstitial tissues. Subsequent $x$-rays were similar. The heart size was normal throughout.

The susceptibility to infections was examined as follows. Tonsillar tissue was present, and lymph nodes were palpable. Biopsy of a small lymph node showed defective follicle formation. Plasma cells were present. The polymorph count always exceeded $4000 / \mathrm{cu} . \mathrm{mm}$. With skin sepsis it increased to over $19,000 /$ cu.mm. Lymphocyte counts exceeded 2770/ cu.mm., except for one count of 1000/cu.mm. associated with a neutrophilia of 11,400 polymorphs/cu.mm. Lymphocyte transformation in response to phytohaemagglutinin was present but probably less than normal, possibly due to the non-specific effect of stress (Riddle and Berenbaum, 1967). In vitro phagocytosis and nitroblue tetrazolium tests were normal (Baehner and Nathan, 1967).

Intradermal monilia antigen gave no response in 48 hours. At 11 weeks the IgG was $96 \mathrm{mg}$. $/ 100 \mathrm{ml}$., the IgA $4 \%$ of reference 'normal' serum, and the IgM 


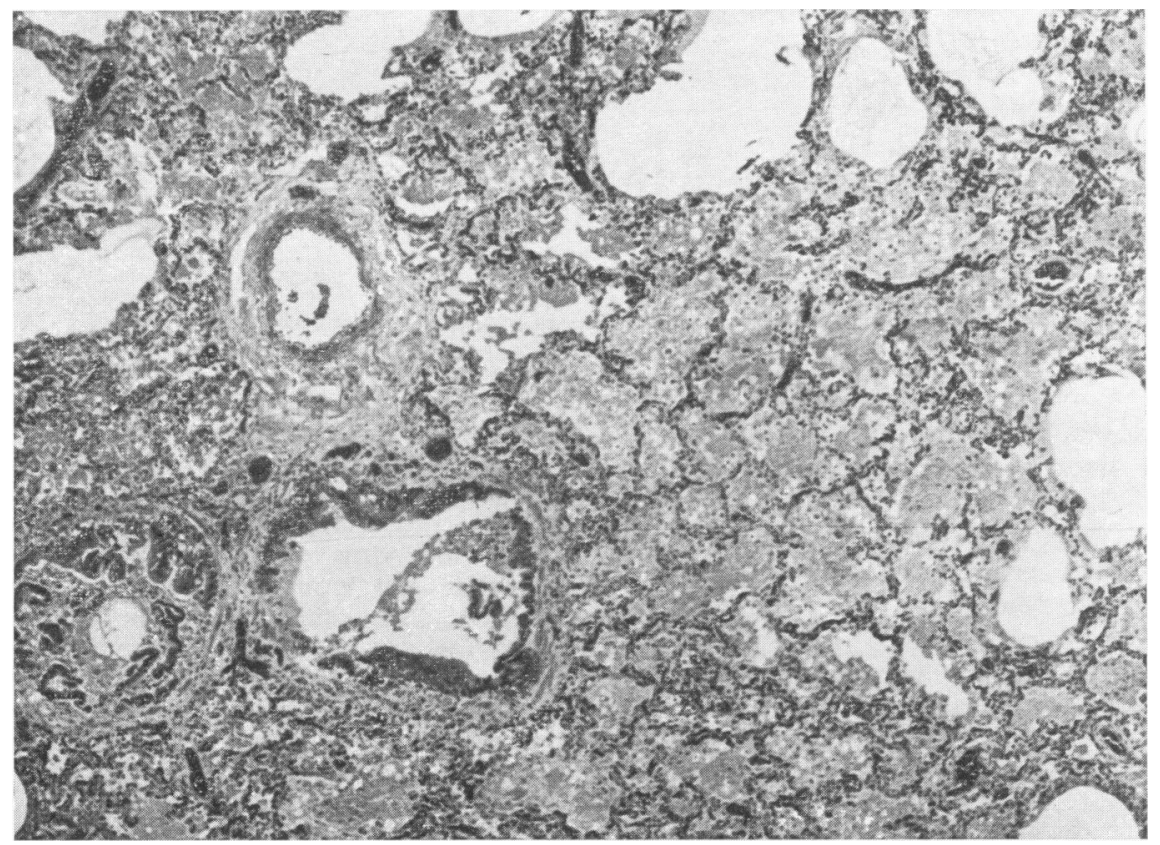

FIG.-A low power photomicrograph of lung, illustrating distension of alveoli with dense, granular, proteinaceous material. Note the absence of septal or alveolar inflammation. (Haematoxylin and eosin. $\times 200$.

$50 \%$ of reference 'normal' serum. At this time he was marasmic, had had diarrhoea for 4 weeks, and had a total serum protein of $4.4 \mathrm{mg} . / 100 \mathrm{ml}$.

The diarrhoea did not respond to diets free of disaccharides, glucose, and galactose. A barium meal and follow-through, stool and urine chromatography, and stool trypsin were normal. Stool cultures grew Esch. coli 0127 on two occasions. This pathogenic Esch. coli in conjunction with prolonged antibiotic therapy were thought partially to explain the prolonged diarrhoea.

In the last 4 days of his illness, he had a raised serum potassium $(5 \cdot 7-7 \cdot 8 \mathrm{mEq} / \mathrm{l}$.) and a low serum sodium (108-117 mEq/1.). The urinary steroid 11-oxygenation index was 2.9 (normal up to 0.7 -Edwards, Makin, and Barratt, 1964) and the 17-ketosteroid excretion was $8 \cdot 8 \mathrm{mg} . / 24 \mathrm{hr}$. (normal below $1 \cdot 0$ ). Clayton, Edwards, and Makin (1969) mentioned this and similar cases in which the high value of the 11-oxygenation index was taken to indicate a partial failure of cortisol biosynthesis in the presence of severe malnutrition.

Necropsy (PM165/68). The body was that of an emaciated white male measuring $58.5 \mathrm{~cm}$. in length and weighing $3470 \mathrm{~g}$. There was purulent exudate about the eyelids and beneath the prepuce. The buttocks were red and macerated. Subcutaneous fat was negligible. There was no clubbing.

The lungs were firm and bulky, and maintained full expansion. Pleural surfaces were normal. Both lungs sectioned with ease, revealing a dry surface. The cut surfaces were irregularly mottled, and had yellow-pink parenchyma contrasting with widespread firm, redbrown confluent patches. The tracheo-bronchial tree was free of aspirated material or exudate. The lungs weighed 82 and $77 \mathrm{~g}$. (average for the age is approximately $35 \mathrm{~g}$. per lung).

The intestines were distended with considerable gas and a small amount of thin liquid material. The mucosa was unremarkable.

The thymus weighed $1 \mathrm{~g}$. Numerous small lymph nodes were present in the mesentery and mediastinum. Tonsils were of normal size. A terminal ileum Peyer's patch was present, though small. The remainder of the gross findings were normal.

Microscopy. All blocks taken from the lungs were similar. There was marked distension of alveoli and some of the terminal branches of the bronchial tree with granular, eosinophilic material containing numerous cholesterol clefts (Fig.). Though there was a resemblance to pulmonary oedema, the material differed from the usual oedema by virtue of its very high protein content-manifested by strong affinity for eosin and PAS reagent-and its very dense granular nature. Furthermore, other features usually associated with pulmonary oedema, such as capillary engorgement, interstitial oedema, and dilatation of perivascular and peribronchial lymphatics were absent.

The material was largely free, but was also present 
within macrophages. These macrophages were large, filled with proteinaceous material, and could be found in various stages of disintegration.

Histochemically, the material was strongly PAS positive, and contained numerous small droplets of sudanophilic lipid. Unstained frozen sections examined in polarized light showed great numbers of cholesterol crystals.

There was no inflammatory infiltrate in either the alveolar septae or alveolar sacs. Pneumocystis carinii organisms were not found, despite careful search.

The intestine showed only a chronic non-specific inflammatory infiltrate in the lamina propria.

The thymus was depleted of lymphocytes, but had numerous Hassal's bodies and plasma cells. Lymph nodes, a Peyer's patch, and appendix also had lymphocytic depletion. Plasma cells were present. The tonsils and spleen were normal.

\section{Comment}

It is of some interest that though he had radiological evidence of pulmonary lesions beginning at approximately 6 weeks of age, he showed no clinical signs of respiratory disease except moderate tachypnoea in the last three days of life. The inappropriately slight pulmonary symptomatology in the presence of extensive radiological and histological disease has been noted in most of the adult cases described. This contrasts with the patients reported by Wilkinson et al. (1968), where both infants showed definite respiratory distress and cough, with subsequent cyanosis in one, and basal rhonchi with subcostal retraction in the other. Their third case (age 23 months) also had cough, dyspnoea, cyanosis, and clubbing.

For most of his life, our patient had persistent superficial infections which resisted therapy, and only partially explained intestinal symptoms. Conventional immune deficiency states and chronic granulomatous disease were considered, but excluded. Though the IgG level was considerably less than 2 standard deviations below the mean reported by Johansson and Berg (1967) for healthy infants of the same age, it was not considered below that expected for an infant with chronic diarrhoea. $\gamma$-globulin was given empirically but without benefit.

The $x$-rays of our patient showed a variable patchy shadowing throughout both lung fields, and a mild air bronchogram effect in only a few films. In contrast, Wilkinson et al. noted a homogeneous, diffuse, perihilar shadowing and prominent air bronchogram effect. The absence of respiratory symptoms except for terminal tachypnoea, in the presence of marked $x$-ray abnormalities suggested an opportunistic infection such as fungal, viral, or Pneumocystis carinii pneumonitis as differential clinical diagnoses. Lung biopsy is usually required to differentiate PAP from these conditions.

Though the clinical and radiological diagnosis of PAP cannot be made in this case (even retrospectively) the histological diagnosis is quite certain. The lungs met the diagnostic criteria presented in the original description; principally, distension of alveoli and distal bronchioles with a granular, acidophilic, strongly PAS positive, lipid material; striking absence of alveolar or interstitial inflammation; and the absence of stainable organisms. Other criteria such as 'sloughed' septal cells, doubly refractile lipid crystals, and laminar bodies were also present.

The histological diagnosis is virtually confined to consideration of Pneumocystis carinii pneumonitis. Though superficial similarities exist, pneumocystis infection is characterized by a reticular 'foamy' exudate, interstitial plasma cell infiltration, and the demonstration of organisms. None of these criteria was present in the sections from this case.

The cause of PAP remains unknown. It has been postulated that the alveolar deposits could represent a response to an infectious agent or to a toxic substance in the environment (Rosen et al., 1958), abnormal production of a substance (surface active agent) normally present in small quantities in the lungs (Larson and Gordinier, 1965), a plasma ultrafiltrate related to inherent defects in pulmonary alveolar capillaries (Taxay, Montgomery, and Wildish, 1960), or to deficient clearance of substances from the lungs (Ramirez, Schultz, and Dutton, 1963; Kuhn et al., 1966; Ramirez and Harlan, 1968).

Rosen et al. (1958) and others have suggested that the source of much of the proteinaceous lipid material is from desquamation and degeneration of proliferating macrophages. This view is suggested by the histological appearance of enlarged macrophages in various stages of degeneration in some of the material. Whether this is a problem of proliferation and overproduction or of deficient disposal has been debated. Recent works favour the latter concept.

Ramirez and Harlan (1968) have presented data strengthening the concept of deficient pulmonary clearance. The lipid component of the exudate in PAP is principally palmitoyl lecithin of pulmonary origin. It is qualitatively similar to that found in other lung diseases, but has none of the surfactant activity attributed to this phospholipid. Synthesis rates of this lipid are normal, further supporting the concept of deficient pulmonary clearance.

Instillation of endobronchial heparin, an agent 
known to activate lipoprotein lipase, has led to the speculations that deficiency of this enzyme was responsible for the alveolar accumulation of lipid. Ramirez and Harlan (1968) suggest that heparinactivated lecithinase may be a more reasonable possibility.

A histologically similar lesion has been produced in laboratory animals. Heppelston (1967) exposed rats to quartz crystal inhalation for periods of 6 to 12 weeks, and instead of producing the classical fibro-nodular histology of pneumoconiosis, the animals developed PAP with retention of large amounts of quartz. The alveolar exudate was interpreted as having been derived from disintegrated macrophages. Gough (1967) showed that rabbits exposed to quartz can produce proteinosis under the special conditions of hyperimmunity to horse serum. Gross and deTreville (1968) exposed rats, guinea-pigs, and hamsters to quartz dust in high concentration. The rats developed proteinosis, and some focal desquamative pneumonitis, while the guinea-pigs and hamsters had extensive desquamative pneumonitis and relatively little proteinosis.

Though the histology of the quartz inhalation model is similar to the human disease, it must be pointed out that there is no reason to believe that the disease in man is necessarily related to inhalation of toxic substances. This was suspected initially because of the occupations of several of the patients, but subsequent case reports and the occurrence of the condition in infancy fail to substantiate this.

\section{Summary}

A third case of pulmonary alveolar proteinosis in infancy is described. This patient had frequent severe extrapulmonary infections and chronic diarrhoea. Radiological onset of pulmonary lesions was as early as 6 weeks, and he died at $3 \frac{1}{2}$ months. In contrast to the previously described cases in infancy the clinical signs of pulmonary disease were negligible relative to the pronounced radiological changes and the widespread histological changes.

The authors wish to thank Professor A. E. Claireaux, The Hospital for Sick Children, Great Ormond Street, London, and Professor Lynne Reid, Brompton Hospital for Chest Diseases, London, for reviewing the histological sections.

\section{REFERENCES}

Baehner, R. L., and Nathan, D. G. (1967). Leukocyte oxidase: defective activity in chronic granulomatous disease. Science, $155,835$.

Clayton, B. E., Edwards, R. W. H., and Makin, H. L. J. (1969). Conditions other than congenital adrenal hyperplasia in which the value of the 11-oxygenation index is raised above normal. F. Endocr., 43, 46p.

Davidson, J. M., and Macleod, W. M. (1969). Pulmonary alveolar proteinosis. Brit. F. Dis. Chest., 63, 13.

Edwards, R. W. H., Makin, H. L. J., and Barratt, T. M. (1964). The steroid 11-oxygenation index: a rapid method for use in the diagnosis of congenital adrenal hyperplasia. f. Endocr., 30, 181 .

Gough, J. (1967). Silicosis and alveolar proteinosis. Brit. med. F., $1,629$.

Gross, P., and deTreville, R. T. P. (1968). Alveolar proteinosis. Arch. Path., 86, 255.

Heppelston, A. G. (1967). Atypical reaction to inhaled silica. Nature (Lond.), 213, 199.

Johansson, S. G. O., and Berg, T. (1967). Immunoglobulin levels in healthy children. Acta paediat. (Uppsala), 56, 572.

Kuhn, C., Györkey, F., Levine, B. E., and Ramirez-Rivera, J. (1966). Pulmonary alveolar proteinosis. Lab. Invest., 15, 492.

Larson, R. K., and Gordinier, R. (1965). Pulmonary alveolar proteinosis. Ann. intern. Med., 62, 292.

Ramirez, J., and Harlan, W. R., Jr. (1968). Pulmonary alveolar proteinosis. Nature and origin of the alveolar lipid. Amer. F. Med., 45, 502.

- Schultz, R. B., and Dutton, R. E. (1963). Pulmonary alveolar proteinosis. Arch. intern. Med., 112, 419.

Riddle, P. R., and Berenbaum, M. C. (1967). Post-operative depression of the lymphocyte response to phytohaemagglutinin. Lancet, $1,746$.

Rosen, S. H., Castleman, B., and Liebow, A. A. (1958). Pulmonary alveolar proteinosis. New Engl. F. Med., 258, 1123.

Taxay, E. P., Montgomery, R. D., and Wildish, D. M. (1960). Studies of pulmonary alveolar microlithiasis and pulmonary alveolar proteinosis. Amer. F. clin. Path., 34, 532.

Wilkinson, R. H., Blanc, W. A., and Hagstrom, J. W. C. (1968). Pulmonary alveolar proteinosis in three infants. Pediatrics, 41, 510 .

Correspondence to the Secretary, Department of Morbid Anatomy, The Hospital for Sick Children, Great Ormond Street, London W.C.1. 